\title{
I point my heart with the tip of my fingers - Biometry for the diagnosis congenital heart defects
}

\author{
Abdulla Tariq ${ }^{\mathrm{a}, \mathrm{b}}$, Dillenseger Jean-Louis ${ }^{\mathrm{a}}$, Summers Ron ${ }^{\mathrm{b}}$, Paul \\ Jean-François $^{\mathrm{c}}$, Houyel Lucile ${ }^{\mathrm{c}}$, Schleich Jean-Marc ${ }^{\mathrm{a}, 1}$, \\ ${ }^{a}$ Inserm, U1099, Rennes, F-35000, France ; Université de Rennes 1, LTSI, Rennes, \\ F-35000, France \\ ${ }^{b}$ Research School of Systems Engineering, Loughborough University, Loughborough, UK \\ ${ }^{c}$ Hôpital Marie-Lannelongue, 92350 Le Plessis Robinson, France \\ ${ }^{d}$ Département de Cardiologie et Maladies Vasculaires, Hôpital de Pontchaillou, Rennes, \\ France
}

\begin{abstract}
Congenital heart defects are characterized by abnormal positioning of some anatomical structures relative to a normal heart. Classically the classification of the disease or the stage of the disease is based on the measurement of the relative position of these structures by the cardiac morphologist. We propose a method in which the heart volume is acquired by a CT scanner. In this paper we present a tool which allows us to define landmarks interactively in this heart volume. These landmarks are then used to estimate some simple geometrical models of the anatomical structures and so to describe their relative position and orientation.

Keywords: Congenital heart disease, heart anatomy, anatomical structures position, interactive landmarks design, geometrical modelling
\end{abstract}

\section{Introduction}

Tetralogy of Fallot is the most common form of cyanotic congenital heart disease. It affects $9 \%-15 \%$ of all infants and children with congenital heart disease (CHD) with an incidence among live births around 2-2.6/10000 and $4 / 10000$ [1] and represents 55-70\% of cyanotic congenital malformations [2]. Tetralogy of Fallot (TOF) belongs to the group of so-called "conotruncal" defects, along with common arterial trunk, malalignment ventricular septal defect (VSD), some types of double outlet right ventricle (DORV), and some 
types of interruption of the aortic arch. This group of CHD is heterogeneous anatomically except for one common anatomic characteristic: the malalignment of the VSD, always located between the two limbs of the septal band.

Knowing these heterogeneous anatomical aspects, it is obviously necessary to define the precise position of all structures forming the heart in order to classify correctly each specimen. Actually the cardiac morphologists perform these anatomical measurements by opening (and so distorting and damaging) the post-mortem heart. Because of the invasiveness of the gesture, the position measurements are relatively difficult to perform. This method also has the disadvantage that the data are difficult to share between several diagnostic centres.

The objective of this study is to design a method for doing accurate congenital heart diagnosis from specimens without opening the heart, using volumes acquired on a 3D image modality.

\section{Material and methods}

\subsection{Key elements of congenital heart diseases}

AA CHD generally causes abnormal positioning of certain cardiac anatomical structures relative to a normal heart. As an example, tetralogy of Fallot can be considered as a failure within the last step of cardiac looping, the wedging, leading to a malalignment between the outlet septum and the rest of the ventricular septum and thus creating the ventricular septal defect. In other words, TOF may result from an arrest of rotation of the outflow tract at the base of the great arteries [3]. As a consequence, the coronary arterial orifices are displaced; the aortic valve is abnormally right-sided, superior and anterior, the pulmonary valve is reciprocally left-sided, inferior and posterior; the position of the aortic and pulmonary valves leaflets is abnormal too, and a VSD by malalignment is created.

Thus, the classification of the disease or the stage of the disease is based on the measurement of the relative position of these structures or group of structures.

The main idea of our study is then to define some anatomical landmarks which will allow us to model the relative position and orientation of the structures involved in the pathology in order to classify the disease. 


\subsection{Landmarks}

The landmarks used for the classification of the disease can be divided into 2 groups:

- One group helps to define the relative orientation and size of the heart. This group includes the apex of the heart, the inferior point of the two ventricular cavities, several points on the interventricular septum, the external junction between the ventricle and the atrium (left and right), and 5 points at the mitral (and tricuspid) valve annulus.

- One group is directly related to the anatomy of the elements involved in characterizing features of the disease: upper tip of the muscular septum, 2 points giving the entrance and orientation of the right and left coronary arteries, 3 points at the pulmonary (and aortic) valve leaflet junctions, and points around the interventricular septal defect.

No fewer than 26 landmarks should be defined within each cardiac volume.

\subsection{Landmarks identification tool}

The main idea of this study is to perform a volume acquisition of the ex-vivo specimen by means of a CT device. The fact that the specimens are ex-vivo allows the acquisition of high resolution CT scans. Several landmarks are identified within this volume. We designed our own volume manipulation and landmark picking tool by combining and developing some plug-ins for the ImageJ package [4]. ImageJ is a useful, lightweight tool, and includes capabilities for image processing, volume loading and 3D visualization facilities. Another advantage of ImageJ is that the plugin architecture makes it easy to integrate new extensions into the software.

However, some specific modules have been modified and some others have been developed especially for picking and visualizing the several required landmarks and handling its list:

- Stack slice viewer. It is the classical way proposed by ImageJ to explore the volume. All the axial slices can be displayed using a slider.

- Orthogonal views. The volume is displayed on the 3 classical orthogonal views (axial sagittal and coronal). On one view, the positions of the 2 orthogonal slices are displayed as a line. A click on any orthogonal view will move the positions of the 2 other orthogonal slices to the $3 \mathrm{D}$ 
position indicated by the click. In the same idea, a click on the stack slice viewer also adjusts the positions of the 3 orthogonal views.

- Landmarks list tool. This tool allows editing, loading or saving the several landmarks picked on the volume. A landmark is characterized by a label and a 3D position given in $\mathrm{mm}$ within the volume. For a specific landmark, a "Show" button allows to display the position of the landmark within the volume stack display tool. A "Mark orthogonal" button will refresh the position of the landmark according to position of the 3 orthogonal views intersection. When the user begins a new project, they can upload a template of landmarks needed for the biometry with a list of labels but with empty locations. However, if needed, new landmarks can be added to the list. The list is saved in a text file which can be processed using a spreadsheet or numerical analysis tool.

- 3D viewer. This tool is based on the classical 3D viewer provided as a plug-in. This tool allows us to display the heart as 3 orthogonal slices in $3 \mathrm{D}$ or as a volume. In this view, the orientation of the heart can be easily modified by the user. The user can also select using a "selection tool" (freehand or other) a part of the volume to cut in order to visualize the inner chambers of the heart. However, some modifications have been provided to the $3 \mathrm{D}$ viewer. First the landmarks can be displayed on the $3 \mathrm{D}$ view as little spheres. The user can pick one sphere with the mouse and move it within the volume. In this case, the sphere remains attached to the surface of the heart. This tool allows us to easily adjust the landmarks positions according to the observed 3D information. Secondly, the model of anatomical information, as the aortic or pulmonary valves annuli, can be displayed on this view.

\subsection{Anatomical information modelling}

From the previous landmarks, geometrical models of the anatomical structures can be defined. For example, under the assumption that the aortic annulus is cylindrical, we can model it as the $3 \mathrm{D}$ circumcircle of the 3 points picked at the valve leaflets junctions. This model gives then a centre, a radius and an orientation. In the same way, the pulmonary valve annulus is also modelled as a circle. From this we have the relative orientations and radii of the pulmonary and aortic valve, giving a statistical measure of the degree of pulmonary valve stenosis. However, the mitral and tricuspid valves are 
not circular, so to provide a closer approximation of their shape, they are modelled with $3 \mathrm{D}$ ellipses. It is for this reason that 5 points are required on both the mitral and tricuspid valves annuli. The ellipses are then estimated by least square fitting. Anatomically the 2 planes supporting the 2 annuli are closely aligned and so serve to define the anatomical plane separating the ventricles and the atrias. The coronary arteries are defined as vectors, by selecting the points where each artery opens into the aorta, and a secondary point within each artery to give the orientation. The interventricular septum is defined as a plane by using a point at the tip of the muscular inventricular septum and 2 other points. Three points (the Apex and the left and right external junctions between atria and ventricles) are used to normalize landmarks for differences in size and rotation between cardiac volumes (procrustes analysis).

\section{Results}

As a preliminary study, volumes of two ex-vivo hearts have been acquired using a Siemens dual source, 128-slice detectors Siemens Somatom CT device. The acquisitions were performed with a high resolution of $0.1504 \times 0.1504 \mathrm{~mm}^{2}$ pixel size within the slice and a $0.1 \mathrm{~mm}$ interslice spacing $(0.6 \mathrm{~mm}$ slice thickness).

Fig. 1 shows a snapshot of the working screen with on the top and partially hidden the stack viewer; on the bottom the 3 orthogonal views (resp. from left to right the axial, the coronal and the sagittal view. The white lines on these views show the location of the other perpendicular views. The window on the left is the landmarks list tool. It shows the label and location of the selected landmarks and the several control buttons. Each window is connected to the others. So a click on one window updates the information in all the windows.

Fig. 2 illustrates the 3D possibilities of the package. The volume can be rendered in order to show the anatomy in 3D (Fig. 2-a). On this view, the user can interactively define a region of interest. The 3D volume behind the region of interest can be discarded in order to look inside the heart cavities. Fig. 2-b shows an example where the cut heart is seen from the top. It allows us to look inside the aorta, the pulmonary artery, the vena cava and the right atrium. The defined landmarks are presented as little spheres. On Fig. 2-c we zoomed on the aorta. This allows us to see the junctions between the aortic valve leaflets. The landmark in front of the annulus is the left 
coronary main artery entrance point. Using the "point selection" tool, the user can add some new landmarks or modify the position of the existing one. In these cases the landmarks list is directly modified.

From these landmarks a first description of the heart is proposed (Fig. 3). The pulmonary valve and aortic valve annuli are modelled as $3 \mathrm{D}$ disks (see also Fig. 2-b and c). The junctions between the leaflets are also incorporated in these models. The centres of these 2 disks define the relative position and orientation of the 2 annuli in the heart. The left and right main coronary arteries orifices are described as $3 \mathrm{D}$ vectors. The mitral valve and tricuspid valve annuli are modelled as $3 \mathrm{D}$ ellipses. The plane containing the mitral valve separates the atrias and the ventricles. It will be used as anatomical landmark for future relative measurement. This is also the case for the interventricular septum plane.

\section{Discusions - Conclusion}

This paper presents the landmarks interactive definition tool which is only the first part of the overall project workflow. However, all the other parts are directly related to this definition tool and its accuracy. From the interactively defined points some heart structures (aortic valve and pulmonary valve annuli, main coronary arteries orifices, the mitral and tricuspid valves annuli,..) have been geometrical defined. The ongoing work is now focused on further geometrical modelling of the others heart structures (freehand surface for the ventricular septal defect) and also on defining a heart referential which will allow us to describe the position and the orientation of the heart structures in a relative normalized referential.

But already we can make some preliminary assessments about this tool. It allows us to perform measurements currently difficult to achieve on cardiac morphologic examination. There are no artefacts associated with incisions of the specimens. And that all the data (original volume but also the landmarks, the model of the structures and the measurements) can be transmitted and therefore are shareable between several medical centres.

\section{References}

[1] M. L. Kirby, Cardiac Development., Oxford University Press, New York, 2007. 
[2] N. Oyen, G. Poulsen, H. A. Boyd, J. Wohlfahrt, P. K. A. Jensen, M. Melbye, Recurrence of congenital heart defects in families., Circulation 120 (4) (2009) 295-301.

[3] M. P. Lomonico, G. W. Moore, G. M. Hutchins, Rotation of the junction of the outflow tract and great arteries in the embryonic human heart., Anat Rec 216 (4) (1986) 544-549.

[4] C. A. Schneider, W. S. Rasband, K. W. Eliceiri, NIH Image to ImageJ: 25 years of image analysis., Nat Methods 9 (7) (2012) 671-675. 


\section{List of Figures}

1 Landmarks list tool, stack viewer (partially hidden) and orthogonal views. The intersection of the 3 orthogonal views points on the right coronary artery. . . . . . . . . . . 9

$23 \mathrm{D}$ view of the outer surface (left), the inner surface after discarding a selected region of interest at the top of the volume (middle) and a zoomed view of the aortic valves (right). The yellow spheres are the selected landmarks. The blue and red disks correspond to respectively the pulmonary valve and aortic valve annuli. . . . . . . . . . . . . . . . .

3 Heart description model: The aortic valve (Ao V.) and pulmonary valve (Pulm V.) annuli; the right (RMCA) and left main coronary arteries entrance points (the left main artery is superimposed over the aortic valve); the mitral valve (Mit V.) and tricuspid valve (Tric V.) annuli; the plane between the ventricles and the atrias; and the interventricular septum plane (IV Sept.). . . . . . . . . . . . . . . . 10 


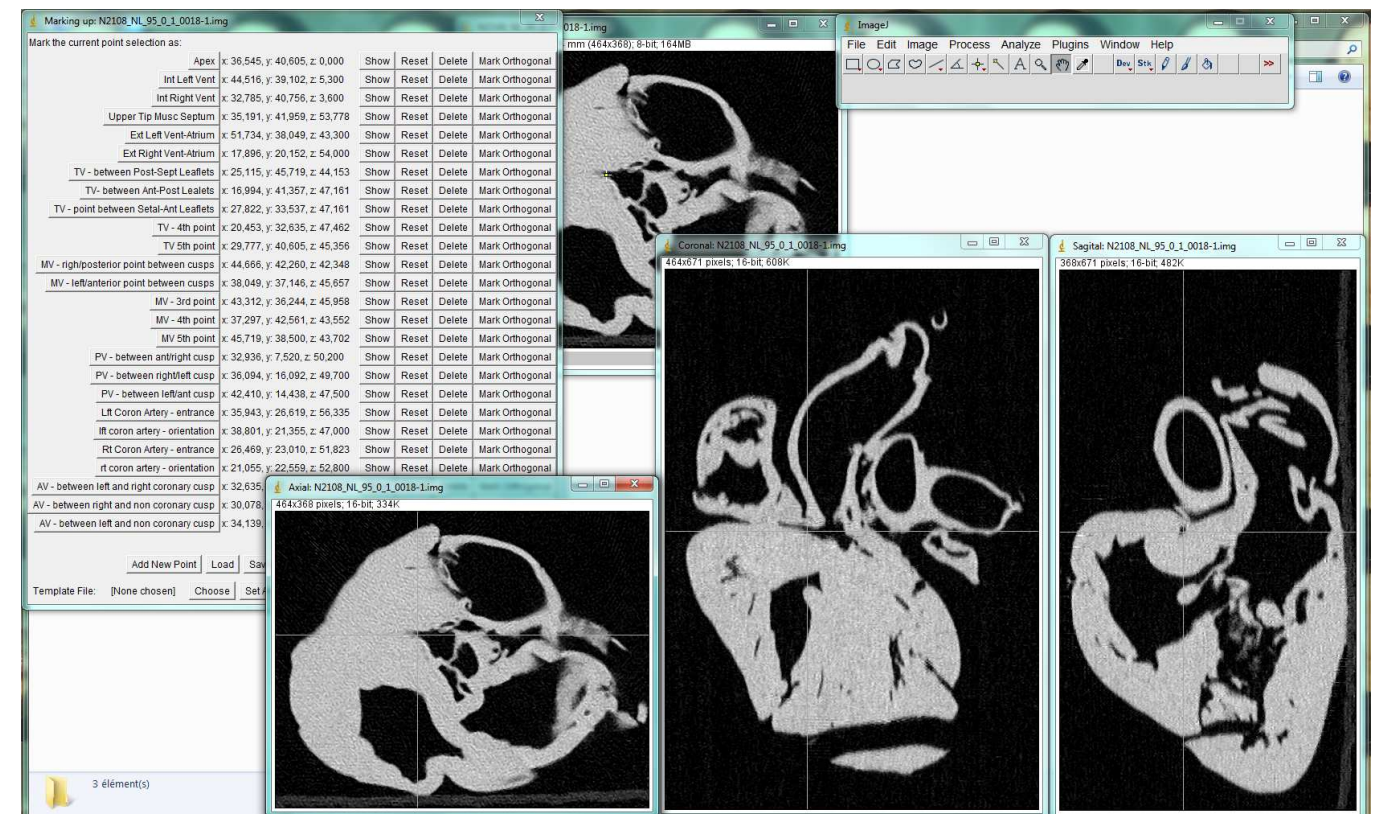

Figure 1: Landmarks list tool, stack viewer (partially hidden) and orthogonal views. The intersection of the 3 orthogonal views points on the right coronary artery.
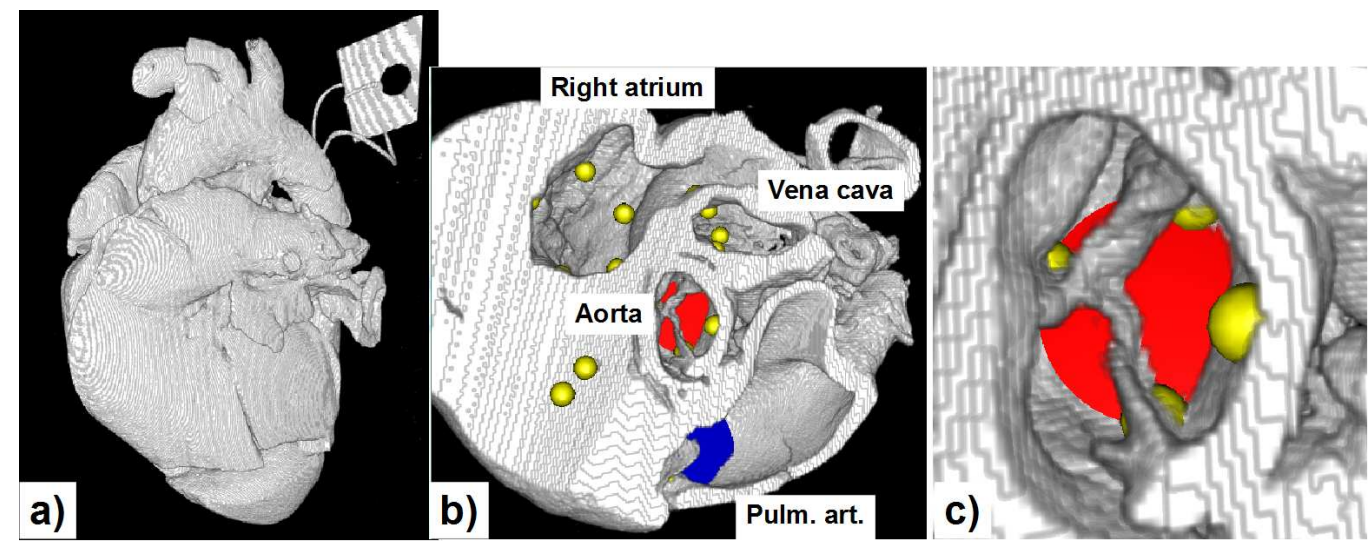

Figure 2: 3D view of the outer surface (left), the inner surface after discarding a selected region of interest at the top of the volume (middle) and a zoomed view of the aortic valves (right). The yellow spheres are the selected landmarks. The blue and red disks correspond to respectively the pulmonary valve and aortic valve annuli. 


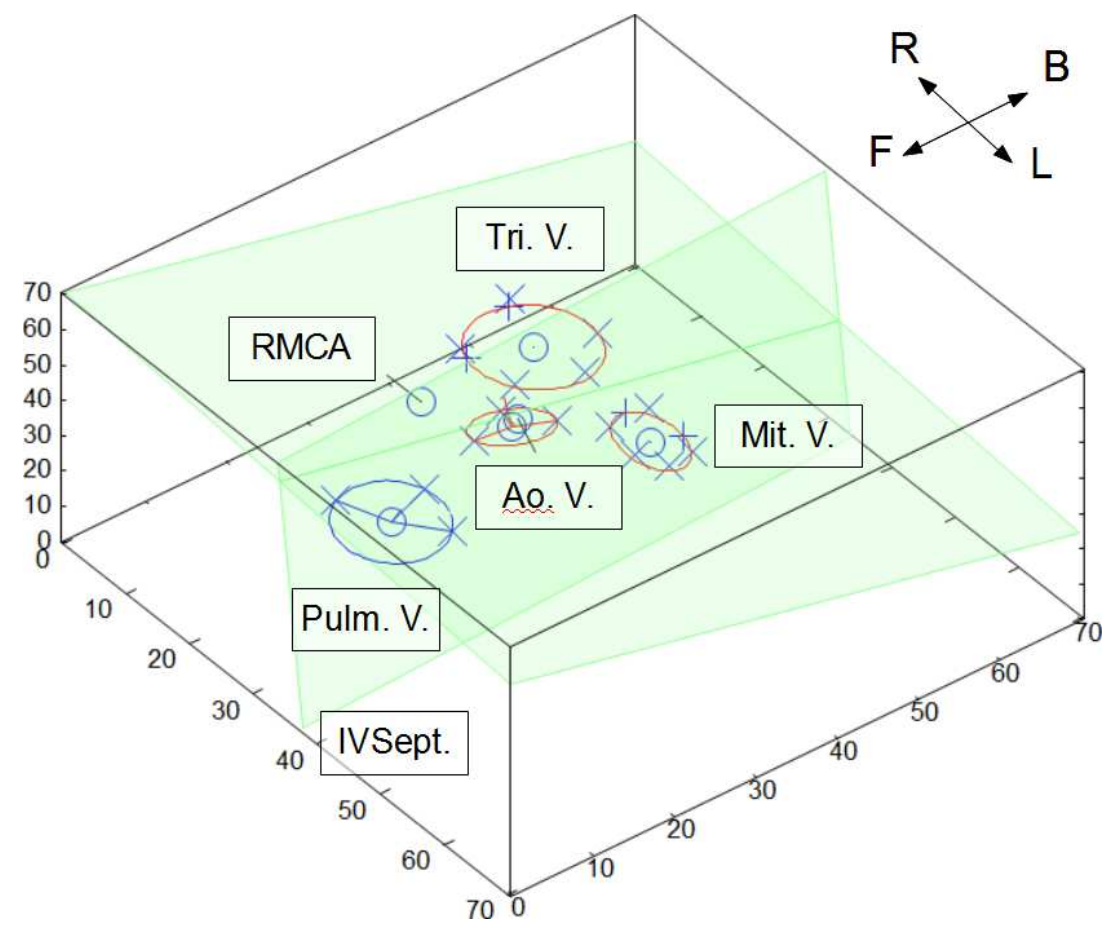

Figure 3: Heart description model: The aortic valve (Ao V.) and pulmonary valve (Pulm V.) annuli; the right (RMCA) and left main coronary arteries entrance points (the left main artery is superimposed over the aortic valve); the mitral valve (Mit V.) and tricuspid valve (Tric V.) annuli; the plane between the ventricles and the atrias; and the interventricular septum plane (IV Sept.). 